\title{
Aspirin resistance in young men with Type 2 diabetes
}

\author{
Subhashini Yaturu ${ }^{1^{*}}$, Usawadee Dier $^{2}$, Huadong Cui ${ }^{2}$, Shaker A. Mousa ${ }^{2}$ \\ ${ }^{1}$ Endocrinology and Metabolism, Stratton VA Medical Center, Albany, USA; \\ *Corresponding Author: Subhashini.Yaturu@va.gov, yaturu@yahoo.com \\ ${ }^{2}$ The Pharmaceutical Research Institute, Albany College of Pharmacy and Health Sciences, Rensselaer, NY, USA \\ Emmy.Dier@acphs.edu, Huadong.Cui@acphs.edu, Shaker.Mousa@acphs.edu
}

Received 29 January 2014; revised 19 February 2014; accepted 25 February 2014

Copyright (C) 2014 Subhashini Yaturu et al. This is an open access article distributed under the Creative Commons Attribution License, which permits unrestricted use, distribution, and reproduction in any medium, provided the original work is properly cited. In accordance of the Creative Commons Attribution License all Copyrights (C) 2014 are reserved for SCIRP and the owner of the intellectual property Subhashini Yaturu et al. All Copyright (C) 2014 are guarded by law and by SCIRP as a guardian.

\section{ABSTRACT}

Objective: Aspirin resistance (AR) or poor response to aspirin is said to be high among subjects with diabetes and more so in patients with poor glycemic control. The aim of our study was to evaluate the prevalence of AR among subjects with diabetes with moderate glycemic control and its association with inflammatory markers and cytokines. Design: This is a single-center open-label prospective clinical study. Methods: The study included 142 young male veterans (mean age of 49 years) with Type 2 diabetes, with HbA1C of $7.7 \pm 1$ 1.1. Urinary 11-dehydro-thromboxane beta-2 (11DhTx2) concentrations measured by immunoassay are the primary outcome measures. Results: Urinary ${ }^{11 D h T x B_{2}}$ levels $\geq 1500$ $\mathrm{pg} / \mathrm{mg}$ of creatinine is considered as AR. Approximately $53 \%$ of subjects had AR. There are no significant differences in the clinical parameters, such as age, history of hypertension or $\mathrm{BMI}$, waist to hip ratio; as well as biochemical parameters, such as HbA1C, lipid parameters or serum creatinine, between subjects with or without AR. Levels of ${ }_{11 D h T x B_{2}} /$ cre correlated with history of CAD, abdominal fat content and IL-6 levels $(p<0.01)$ as well as abdominal fat content and IL- 6 levels; duration of diabetes and history of CAD. Conclusions: Aspirin resistance is common in subjects with diabetes even with moderate control. Additional measures to improve aspirin response should be considered. Further studies with larger groups are needed to clarify the AR with various associated risk fac- tors.

\section{KEYWORDS}

Aspirin; Aspirin Resistance; Type 2 Diabetes; Men; CRP; IL-6; Adiponectin; Body Composition; Abdominal Fat

\section{BACKGROUND}

Prevalence of diabetes is increasing rapidly worldwide. Diabetes is projected to affect 300 million people around the world by 2025. Type 2 diabetes (T2DM) is the most common form of diabetes. Patients with T2DM carry an equivalent cardiovascular risk to that of a non-diabetic individual who has already experienced a coronary event. Platelets play an important role in atherogenesis and its thrombotic complications in diabetic patients. Long-term aspirin administration in patients at high risk of occlusive vascular events reduced up to 34\% of nonfatal myocardial infarction (MI), $25 \%$ of nonfatal stroke, and $18 \%$ of all-cause mortality [1]. The American Diabetes Association (ADA) recommends enteric-coated aspirin at a dosage of 81 - $325 \mathrm{mg}$ to be used as a preventive strategy in high-risk diabetic individuals [2], including those who are over 40 years of age or who have additional risk factors (family history of CVD, hypertension, smoking, dyslipidemia, or albuminuria). Low-dose aspirin (as low as $81 \mathrm{mg}$ ) irreversibly inhibits the COX-1 enzyme, by acetylating the serine residue at position 529, consequently impairing the transformation of arachidonic acid to prostaglandin (G2/H2), and thromboxane (Tx) A2, which is a potent mediator of platelet aggregation and activation. This effect explains the clinical benefit of aspirin in patients with high risk vascular disease [3]. Aspirin is ineffective in a large proportion of the popula- 
tion: a problem termed "aspirin resistance". Aspirin resistance (AR), defined as failure of suppression of thromboxane generation, increases the risk of cardiovascular events in a high-risk population [4]. Causes of aspirin resistance include concurrent use of nonsteroidal anti-inflammatory drugs, such as ibuprofen that may compete with aspirin at the COX-1 receptor site [5], polymorphisms in the COX-1 gene [6], poor glucose control, body weight and conditions associated with a high platelet turnover [7-10]. Objective of our study was to evaluate the prevalence of AR among subjects with moderate control and its association with inflammatory markers and cytokines.

\section{MATERIALS AND METHODS}

This is a single-center open-label prospective clinical study. The clinical study part was carried out at Overton Brooks VA Medical Center (VAMC) at Shreveport, Louisiana after the approval of the protocol and consent form by the Institutional Review Board at LSUHSC, Shreveport as well as the Research and Development Committee at VAMC. All the subjects have provided informed consent. The samples for this clinical study were collected at Overton Brooks VAMC, Shreveport, LA between 2006 and 2009. Plasma from the venous blood samples drawn for insulin, C-reactive protein (CRP), tumor necrosis factor $\alpha$ (TNF-a), interleukin-6 (IL-6) and adiponectin and urine samples were stored at $-80^{\circ} \mathrm{C}$ until further analysis. For the study of aspirin resistance, supplemental analyses of the samples were carried out at Stratton VAMC in Albany, NY and at the Pharmaceutical Research Institute at Albany College of Pharmacy and Health Sciences in Rensselaer, NY. The inclusion criteria included men aged 30 - 55 years of all ethnic groups with Type 2 diabetes and normal kidney function, evaluated by creatinine $<1.4$ and eGFR $>70$. One hundred and forty two subjects with Type 2 diabetes were included in the study. Clinical parameters include weight, height, waist circumference, duration of diabetes, history of hypertension, and history of premature coronary artery disease. Basic labs collected include fasting lipids, HbA1C, uric acid, serum creatinine and urine micro-albumin in all subjects. Body composition analysis was carried out by DXA.

Laboratory measurements: Levels of total adiponectin, MCP, TNF- $\alpha$, CRP, IL-6 and insulin were measured in duplicate using commercially available kits (ALPCO Diagnostics, USA). The sensitivity of the adiponectin assay is $0.234 \mathrm{ng} / \mathrm{mL}$ (range of $0.375-12 \mathrm{ng} / \mathrm{mL}$ ). Interand intra-assay coefficients of variation were $2.8 \%$ $5.5 \%$ and $2.97 \%-3.84 \%$, respectively. The sensitivity of the CRP assay is $0.124 \mathrm{ng} / \mathrm{mL}$. Inter- and intra-assay coefficients of variation were $5.5 \%-6 \%$ and $11 \%-13 \%$, respectively. The sensitivity of the TNF- $\alpha$ assay is 4.8 $\mathrm{pg} / \mathrm{mL}$. Inter- and intra-assay coefficients of variation were $\pm 10.8 \%$ and $4 \%-8.3 \%$, respectively. The sensitivity of the insulin assay is $1 \mu \mathrm{IU} / \mathrm{mL}$. Inter- and intra-assay coefficients of variation were $2.6 \%-3.6 \%$ and $2.8 \%$ $4 \%$, respectively. The insulin resistance index was calculated from fasting glucose and insulin using the formula of homeostatic model assessment (HOMA-R). HOMA-R $=$ (plasma glucose level $\mathrm{x}$ plasma insulin level/22.5). Urinary 11-dehydro-thromboxane beta-2 (11DhTx2) concentrations were measured using an enzyme immunoassay kit from Cayman Chemicals with a specificity of $100 \%$ and sensitivity at $120 \mathrm{pg} / \mathrm{ml}$ and an intra- and interassay variability of $5 \%-7 \%$. Levels of $11 \mathrm{DhTx} 2$, a major urinary metabolite of thromboxane at $\geq 1500 \mathrm{pg} / \mathrm{mg}$ of creatinine is considered as AR.

Aspirin: Bioassay of aspirin in the serum was carried out by Liquid Chromatography Mass Spectrometry (LC/MS/MS) in MRM mode. LC/MS/MS in MRM mode offers analytical specificity superior to that of immunoassay or conventional HPLC for low molecular weight analyses. Aspirin levels are expressed as $\mu \mathrm{g} / \mathrm{mL}$ and are the sum of salicylic acid, which were eluted at different times as various glucoronides possess different retention times.

Statistical analyses: Statistical analyses were carried out using Microsoft Excel 2007. All data are presented as means \pm S.D. A $p$ value of $<0.05$ is considered statistically significant. Difference in the clinical and biochemical parameters between the subjects with elevated 11DhTx2/cre levels and those with normal levels was carried out. Correlation of 11DhTx2/cre levels with other clinical and biochemical parameters were carried out.

\section{RESULTS}

Baseline characteristics of the cohort are shown in Table 1. Participants included 142 male patients with Type 2 diabetes. Their average age was 48 years, and most were Caucasian. The levels of insulin, HbAIC, and adipocytokines are shown in Table 2. Approximately $55 \%$ of subjects had aspirin resistance (poor response to aspirin) as measured by elevated urinary 11-dehydrothromboxane beta- $2\left(11 \mathrm{DhTx}_{2}\right)$ concentration. The comparative clinical parameters of the subjects with T2DM with or with aspirin resistance are shown in Table 3.

Compared to the subjects with $11 \mathrm{DhTx}_{2} /$ cre levels < 1500 , there are no significant differences in the clinical parameters such as age, history of hypertension or BMI, waist to hip ratio.

Compared to the subjects with $11 \mathrm{DhTx}_{2} /$ cre levels < 1500 , there are no significant differences in the biochemical parameters such as creatinine, thyroid function tests, HbA1C and lipid parameters. Levels of 11DhTx2/ cre correlated with $\%$ abdominal fat $(r=0.19 ; p<0.01)$, and IL-6 levels $(r=0.18 ; p<0.01)$. Elevated 11DhTx2/ 
cre levels not associated with the other adipocytokines, duration of diabetes, use of insulin or micro-albumin.

Table 1. Clinical characteristics of subjects with premature coronary artery disease.

\begin{tabular}{cc}
\hline & Mean \pm SD \\
\hline Age & $49 \pm 3$ \\
BMI & $31 \pm 6.4$ \\
Creatinine & $1.05 \pm 0.16$ \\
GFR & $81.5 \pm 15$ \\
HTN (\%) & $85 \%$ \\
DM (\%) & $40 \%$ \\
PAD & $5 \%$ \\
Current smoker & $21 \%$ \\
\% Abdominal fat & $34.7 \pm 1.8$ \\
\hline
\end{tabular}

DM: diabetes; BMI: basal metabolic index; GFR: glomerular filtration rate; HTN: hypertension; PAD: peripheral arterial disease

Table 2. Biochemical and adipokine parameters in subjects with Type 2 diabetes.

\begin{tabular}{cc}
\hline Parameter & Mean \pm SD \\
\hline Insulin & $13.5 \pm 14$ \\
HbA1C & $7.7 \pm 1.1$ \\
MCP-1 & $184 \pm 99$ \\
CRP & $4.5 \pm 0.76$ \\
IL-6 & $7.6 \pm 1.7$ \\
TNF- $\alpha$ & $14.8 \pm 2.3$ \\
Visfatin & $2.09 \pm 0.55$ \\
\hline
\end{tabular}

MCP-1: Monocyte chemo attractant protein-1; CRP: C-reactive protein; IL-6: interleukin-6; TNF- $\alpha$ : tumor necrosis factor $\alpha$.

Table 3. Comparison of clinical parameters in subjects with Type 2 DM with or without aspirin resistance.

\begin{tabular}{lccc}
\hline & No AR & AR & $\boldsymbol{p}$-value \\
\hline TxB2 & $978 \pm 35$ & $3801 \pm 285$ & $3.75 \mathrm{E}-15$ \\
Age & $49 \pm 0.6$ & $48 \pm 0.7$ & 0.322977 \\
BMI & $34 \pm 0.6$ & $33.9 \pm 0.6$ & 0.762581 \\
Waist/Hip & $1.05 \pm 0.05$ & $1.05 \pm 0.05$ & NS \\
DM Duration & $7.1 \pm 0.7$ & $8.1 \pm 0.7$ & 0.294201 \\
HTN & 89 & 87 & NS \\
CAD (\%) & 25 & 21 & NS \\
\hline
\end{tabular}

AR: aspirin resistance; TxB2: thromboxane B2 Clearance rate; BMI: Body mass index; waist/hip: waist to hip ratio; HTN: hypertension; CAD: coronary artery disease.

\section{DISCUSSION}

Individuals with diabetes are at two- to fourfold increased risk of cardiovascular events compared with ageand sex-matched individuals without diabetes. In diabetic patients over the age of 65 years, $68 \%$ of deaths are from coronary heart disease (CHD) and 16\% are from stroke. A position statement of the American Diabetes Association, a scientific statement of the American Heart Association, and an expert consensus document of the American College of Cardiology Foundation recommends enteric-coated aspirin at a dosage of 81 - $325 \mathrm{mg}$ to be used as a preventive strategy in high-risk diabetic individuals [2]. Despite the proven benefits of aspirin therapy in the prevention of cardiovascular complications in the diabetic patient, the diabetic patient has a greater risk for thrombotic complications than non-diabetic patients.

In a sub-analysis of the Japanese Primary Prevention of Atherosclerosis with Aspirin for Diabetes (JPAD) trial to evaluate the effect of low-dose aspirin on preventing atherosclerotic events in groups receiving different diabetes management, the study noted that low-dose aspirin (81 or $100 \mathrm{mg}$ daily) reduced atherosclerotic events predominantly in the diet-alone group but not in the insulin or oral hypoglycemic agent user groups [11]. They also noted that patients treated with insulin had the longest history of diabetes, the worst glycemic control, and the highest prevalence of diabetic micro angiopathies and low dose aspirin did not affect atherosclerotic events (insulin: hazard ratio [HR] 1.19 [95\% CI 0.60-2.40]).

In our study, we found that AR is significantly high. Platelet aggregation depends upon several factors. Aspirin effect truly on generation of thromboxane. Hence measuring metabolites of thromboxane is appropriate. Most of the studies used platelet aggregometry to measure AR, which makes it difficult to compare our results with previous studies.

In an analysis from the aspirin-induced platelet effect (ASPECT) study, diabetic patients treated with $81 \mathrm{mg}$ exhibited higher platelet function measured by VerifyNow, collagen- and ADP-induced LTA, and 11-dh-TxB(2) levels ( $p<$ or $=0.02$ for all comparisons). Higher aspirin doses significantly inhibited platelet function and decreased aspirin resistance in diabetic patients $(p<0.05)$ [12].

\section{URINARY 11-dh-TxB2}

We noted that subjects with coronary artery disease have higher levels of 11 DhTxB2 levels. Our results are similar to the results in ASPECT study, where they reported that diabetic patients with CAD treated with 81 mg aspirin exhibit a higher prevalence of aspirin resistance and have significantly higher ADP- and collagen-induced platelet aggregation, 11-dh-TxB(2) levels [12]. They also noted that both 162 and $325 \mathrm{mg}$ aspirin significantly reduced levels compared with $81 \mathrm{mg}$ aspirin in diabetic patients ( $p=0.01$ and 0.002 , respectively).

On-going studies that will address the problem include: A Study of Cardiovascular Events in Diabetes (ASCEND), being conducted in the U.K., has recruited 15,000 people 
with diabetes (either Type 1 or Type 2) who were not known to have vascular disease. ASCEND volunteers are randomly allocated to take either $100 \mathrm{mg}$ aspirin daily or placebo (dummy) and 1 gram capsules containing naturally occurring omega-3 fatty acids ("fish-oils") or placebo capsules containing olive oil.

http://www.ctsu.ox.ac.uk/ascend/. The trial started during 2004, and is scheduled to continue until 2017. The other study is Aspirin and Simvastatin Combination for Cardiovascular Events Prevention Trial in Diabetes (ACCEPT-D). It is an open-label Italian primary prevention trial assessing whether $100 \mathrm{mg}$ /daily of aspirin prevents cardiovascular events in patients without clinically manifest vascular disease and treated with simvastatin (starting dose $20 \mathrm{mg} /$ day) comparing to no aspirin among adults over age 50 years with diabetes [13].

Statins to improve aspirin resistance: There is evidence that statins may be useful in treating aspirin resistance. Tirnaksiz and associates reported in a study of subjects with stable coronary artery disease, $11.2 \%$ were found to be aspirin resistant as measured by PFA-100, with a closure time of $<186$ seconds with collagen/ adrenaline cartridges [14]. After 3 months of statin therapy (atorvastatin $10 \mathrm{mg} /$ day), 65\% of the aspirin-resistant patients became aspirin sensitive by PFA-100 measurements ( $p<0.0001)$ [14]. Tekten and his colleagues have shown that statins reduced platelet aggregation [15].

Other suggested recommendations include: As saturated fat ingestion increases in vivo thromboxane production despite aspirin therapy, low dietary saturated fat intake and aggressive lipid management is recommended in diabetic patients on ASA therapy [16].

\section{CONCLUSIONS}

Aspirin resistance is high with $81 \mathrm{mg}$ a day of aspirin among subjects with diabetes, who are at high risk for atherothrombotic episodes. Screening for aspirin resistance and alternative methods to improve response in those with AR are suggested.

\section{ACKNOWLEDGMENTS}

The residual funds from the Merit Review grant of Dr. Yaturu partly supported the study. Part of the support is by The Pharmaceutical Research Institute at the Albany College of Pharmacy and Health Sciences, Rensselaer, NY. Dr. Yaturu receives salary support from VHA.

The authors appreciate the proof reads by Barbara Youngberg.

\section{REFERENCES}

[1] (2002) Collaborative meta-analysis of randomised trials of antiplatelet therapy for prevention of death, myocardial infarction, and stroke in high risk patients. $B M J, 324,71-$ 86. http://dx.doi.org/10.1136/bmj.324.7329.71
[2] Pignone, M., et al. (2010) Aspirin for primary prevention of cardiovascular events in people with diabetes: A position statement of the American Diabetes Association, a scientific statement of the American Heart Association, and an expert consensus document of the American College of Cardiology Foundation. Diabetes Care, 33, 13951402. http://dx.doi.org/10.2337/dc10-0555

[3] Rao, G.H. (1987) Influence of anti-platelet drugs on platelet-vessel wall interactions. Prostaglandins, Leukotrienes and Medicine, 30, 133-145. http://dx.doi.org/10.1016/0262-1746(87)90143-0

[4] Eikelboom, J.W., et al., (2002) Aspirin-resistant thromboxane biosynthesis and the risk of myocardial infarction, stroke, or cardiovascular death in patients at high risk for cardiovascular events. Circulation, 105, 1650-1655. http://dx.doi.org/10.1161/01.CIR.0000013777.21160.07

[5] Catella-Lawson, F., et al., (2001) Cyclooxygenase inhibitors and the antiplatelet effects of aspirin. The New England Journal of Medicine, 345, 1809-1817. http://dx.doi.org/10.1056/NEJMoa003199

[6] Halushka, M.K. and Halushka, P.V. (2002) Why are some individuals resistant to the cardioprotective effects of aspirin? Could it be thromboxane A2? Circulation, 105, 1620-1622. http://dx.doi.org/10.1161/01.CIR.0000015422.86569.52

[7] Modica, A., Karlsson, F. and Mooe, T. (2007) Platelet aggregation and aspirin non-responsiveness increase when an acute coronary syndrome is complicated by an infection. Journal of Thrombosis and Haemostasis, 5, 507-511. http://dx.doi.org/10.1111/j.1538-7836.2007.02378.x

[8] Guthikonda, S., et al. (2007) Reticulated platelets and uninhibited COX-1 and COX-2 decrease the antiplatelet effects of aspirin. Journal of Thrombosis and Haemostasis, 5, 490-496.

http://dx.doi.org/10.1111/j.1538-7836.2007.02387.x

[9] Zimmermann, N., et al. (2005) Is cardiopulmonary bypass a reason for aspirin resistance after coronary artery bypass grafting? European Journal Cardio-Thoracic Surgery, 27, 606-610. http://dx.doi.org/10.1016/j.ejcts.2004.12.010

[10] Zimmermann, N., et al. (2003) Functional and biochemical evaluation of platelet aspirin resistance after coronary artery bypass surgery. Circulation, 108, 542-547. http://dx.doi.org/10.1161/01.CIR.0000081770.51929.5A

[11] Okada, S., et al. (2011) Differential effect of low-dose aspirin for primary prevention of atherosclerotic events in diabetes management: A subanalysis of the JPAD trial. Diabetes Care, 34, 1277-1283. http://dx.doi.org/10.2337/dc10-2451

[12] DiChiara, J., et al. (2007) The effect of aspirin dosing on platelet function in diabetic and nondiabetic patients: an analysis from the aspirin-induced platelet effect (ASPECT) study. Diabetes, 56, 3014-3019. http://dx.doi.org/10.2337/db07-0707

[13] De Berardis, G., et al. (2007) Aspirin and Simvastatin Combination for Cardiovascular Events Prevention Trial in Diabetes (ACCEPT-D): Design of a randomized study of the efficacy of low-dose aspirin in the prevention of cardiovascular events in subjects with diabetes mellitus 
treated with statins. Trials, $8,21$.

http://dx.doi.org/10.1186/1745-6215-8-21

[14] Tirnaksiz, E., et al. (2009) Effect of high dose statin therapy on platelet function; statins reduce aspirin-resistant platelet aggregation in patients with coronary heart disease. Journal of Thrombosis and Haemostasis, 27, 2428. http://dx.doi.org/10.1007/s11239-007-0154-1
[15] Tekten, T., et al. (2004) The effect of atorvastatin on platelet function in patients with coronary artery disease. Acta Cardiologica, 59, 311-315. http://dx.doi.org/10.2143/AC.59.3.2005187

[16] Yassine, H.N., et al. (2010) Effect of glucose or fat challenge on aspirin resistance in diabetes. International Journal of Endocrinology, 2010, Article ID: 820876. 